\title{
QUASICONFORMAL EXTENSIONS OF MAPPINGS IN $n$-SPACE
}

\author{
DAVID B. GAULD and M. K. VAMANAMURTHY
}

\begin{abstract}
Let $D$ be a Jordan domain in $\overline{\mathbf{R}}^{n}$. Then a homeomorphism $h: \partial D \rightarrow$ $\rightarrow S^{n-1}$ extends to a homeomorphism $\hat{h}: \bar{D} \rightarrow \bar{B}^{n}$ so that $\hat{h} \mid D$ is quasiconformal if and only if each point of $\partial D$ has a neighbourhood in $\bar{D}$ over which $h$ extends quasiconformally. One consequence of this is a quasiconformal version of the generalised Schoenflies theorem. Another consequence of the methods is that if $D$ is a domain in $\overline{\mathbf{R}}^{n}$ and $h: \partial D \rightarrow \bar{B}^{n-1}$ is a homeomorphism, then $h$ extends to a quasiconformal homeomorphism of $\overline{\mathbf{R}}^{n}$ if and only if each point of $\partial D$ has a neighbourhood over which $h$ extends quasiconformally.
\end{abstract}

1. The main results. This paper consists essentially of two parts. The major result in the first part is Theorem 1 concerning the flattening of a locally flat boundary. As the result is already known in the topological case, it is stated and proved in the quasiconformal context, the proof being an adaptation of that of Connelly [3].

The major result of the second part is Theorem 3 which concerns the structure of the interior of a set given that the boundary is already nice. The technique used to prove Theorem 3 is a modification of Brown's proof in [2] of the generalised Schoenflies theorem. As observed in [14, p. 139, Remark 1], Brown's proof of the generalised Schoenflies theorem makes use of non-quasiconformal homeomorphisms. We modify his proof using a technique developed in [1] and [5] and we make use of only quasiconformal auxiliary homeomorphisms. Hopefully the technique will be of use elsewhere.

Please refer to $\S 2$ for the definitions and notation used in the following results.

Theorem 1. Suppose $D$ is a Jordan domain in $\overline{\mathbf{R}}^{n}$ and that $\partial D$ is locally quasiconformally flat in $D$. Then $\partial D$ is quasiconformally flat in $D$.

A two-sided version of Theorem 1 is formulated in $\S 6$.

In the case where the domain $D$ is non-Jordan, we can still formulate a suitable 
definition of (local) quasiconformal flatness as well as a more general version of Theorem 1: details appear in $\S 4$. Also in $\S 4$ we prove the following result.

Theorem 2. Suppose $X$ is a subset of $\overline{\mathbf{R}}^{n}$ and $h: X \rightarrow \bar{B}^{n-1}$ is a homeomorphism. Suppose further that $\mathscr{U}$ is a cover of $X$ by open subsets of $\overline{\mathbf{R}}^{n}$ so that for each $U \in \mathscr{U}, h \mid U \cap X$ extends to a quasiconformal embedding $h_{U}: U \rightarrow \overline{\mathbf{R}}^{n}$. Then $h$ extends to a quasiconformal embedding of a neighbourhood of $X$ in $\overline{\mathbf{R}}^{n}$.

Theorem 3. Let $e: N \rightarrow \overline{\mathbf{R}}^{n}$ be an embedding where $N$ is a neighbourhood of $S^{n-1}$ in $\bar{B}^{n}$. Then e| $S^{n-1}$ extends to an embedding $\hat{e}: \bar{B}^{n} \rightarrow \overline{\mathbf{R}}^{n}$ with $e(N) \subset \hat{e}\left(\bar{B}^{n}\right)$. If $e \mid N \cap B^{n}$ is quasiconformal, then so is $\hat{e} \mid B^{n}$.

Theorem 3 is Gehring's Theorem 1 in [6], but whereas Gehring uses the ideas of Mazur [10], we adapt the ideas of Brown's completely different proof [2].

Corollary 4. A Jordan domain $D$ in $\overline{\mathbf{R}}^{n}$ is quasiconformally equivalent to $B^{n}$ if and only if $\partial D$ is locally quasiconformally flat in $D$.

Corollary 5. Suppose that $D$ is a domain in $\overline{\mathbf{R}}^{n}$ whose boundary, $\partial D$, is homeomorphic to the $(n-1)$-ball $\bar{B}^{n-1}$ by a homeomorphism $h: \partial D \rightarrow \bar{B}^{n-1}$ so that for each point $x$ of $\partial D, h$ extends to a quasiconformal embedding of a neighbourhood (in $\overline{\mathbf{R}}^{n}$ ) of $x$ in $\overline{\mathbf{R}}^{n}$. Then $h$ extends to a quasiconformal homeomorphism of $\overline{\mathbf{R}}^{n}$. In particular, $D$ is quasiconformally equivalent to $B^{n}$.

Remark. For $n=3$, a slightly stronger version of Corollary 5 holds: see [7], [11].

Corollary 6. Let $e: S^{n-1} \rightarrow \overline{\mathbf{R}}^{n}$ be an embedding: thus by the Jordan-Brouwer separation theorem $[9, p .80] \overline{\mathbf{R}}^{n}-e\left(S^{n-1}\right)$ consists of two components, each being a Jordan domain. Suppose that $e^{-1}$ is locally quasiconformally flat in each of these Jordan domains. Then e extends to a quasiconformal homeomorphism of $\overline{\mathbf{R}}^{n}$.

Corollary 6 is a quasiconformal version of the generalised Schoenflies theorem. Of course, one can delete all mention of quasiconformality in the above results (except the 'only if' part of Corollary 4) and still have valid results (cf. Remark 1 on p. 139 of [14]).

2. Preliminaries. The following notation is used. $\mathbf{R}^{n}$ denotes euclidean $n$-space with the Pythagorean metric: if $x=\left(x_{1}, \ldots, x_{n}\right) \in \mathbf{R}^{n}$, let $\|x\|=\left(x_{1}^{2}+\ldots+x_{n}^{2}\right)^{1 / 2}$; then the distance from $x$ to $y$ in $\mathbf{R}^{n}$ is $\|x-y\| . \overline{\mathbf{R}}^{n}$ denotes the one-point compactification of $\mathbf{R}^{n} ; \overline{\mathbf{R}}^{n}=\mathbf{R}^{n} \cup\{\infty\}$. If $x \in \mathbf{R}^{n}, r \in \mathbf{R}$, then $r x \in \mathbf{R}^{n}$ is obtained by multiplying each coordinate of $x$ by $r$.

For $a, b \in \mathbf{R}$ with $0 \leqq a \leqq b$, let

$$
\begin{aligned}
& {[a, b]=\left\{x \in \mathbf{R}^{n} \mid a \leqq\|x\| \leqq b\right\}} \\
& (a, b)=\left\{x \in \mathbf{R}^{n} \mid a<\|x\|<b\right\},
\end{aligned}
$$


with obvious analogous meanings for $[a, b)$ and $(a, b]$. In particular, $[a, a]=[a]$, $[0,1)=B^{n},[0,1]=\bar{B}^{n}$ and $[1,1]=[1]=S^{n-1}$.

Intervals in $\mathbf{R}$ will be denoted by $I[a, b], I(a, b)$, etc.

If $X$ is any subset of $\overline{\mathbf{R}}^{n}$, we let $\bar{X}$, int $(X)$ and $\partial X$ denote, respectively, the closure, interior and frontier of $X$, all with respect to $\overline{\mathbf{R}}^{n}$. A domain is a nonempty connected open subset of $\overline{\mathbf{R}}^{n}$, a Jordan domain being a domain in $\overline{\mathbf{R}}^{n}$ whose frontier is homeomorphic to $S^{n-1}$. We use the words "embedding" and "homeomorphism" in their usual topological senses: homeomorphisms must be surjective.

Let $h: D \rightarrow D^{\prime}$ be a homeomorphism between two domains in $\overline{\mathbf{R}}^{n}$. Then $h$ is quasiconformal if and only if

$$
\left\{\limsup _{r \rightarrow 0} \frac{\max _{\|x-y\|=r}\|h(x)-h(y)\|}{\min _{\|x-y\|=r}\|h(x)-h(y)\|} \mid x \in D-\left\{\infty, h^{-1}(\infty)\right\}\right\}
$$

is bounded. Other equivalent definitions of quasiconformality appear in [14].

Definition. Let $D$ be a Jordan domain in $\overline{\mathbf{R}}^{n}$ and $h: \partial D \rightarrow S^{n-1}$ a homeomorphism. Then $h$ is locally quasiconformally flat in $D$ if and only if there is a real number $a, 0<a<1$, and a cover $\mathscr{U}$ of $\partial D$ by open subsets of $\bar{D}$ so that for each $U \in \mathscr{U}$, the map $h \mid U \cap \partial D$ extends to a homeomorphism

$$
h_{U}: \bar{U} \rightarrow\left\{r z \in \mathbf{R}^{n} \mid a \leqq r \leqq 1 \text { and } z \in h(\bar{U} \cap \partial D)\right\}
$$

so that $h_{U} \mid U \cap D$ is quasiconformal.

We say that $h$ is quasiconformally flat in $D$ if and only if the cover $\mathscr{U}$ above may be chosen to contain just one element.

The boundary $\partial D$ is (locally) quasiconformally flat in $D$ if and only if there exists a homeomorphism $h: \partial D \rightarrow S^{n-1}$ which is (locally) quasiconformally flat.

We now comment on the above definition. The ideal situation would not assume the local extendability of a global homeomorphism $h: \partial D \rightarrow S^{n-1}$; cf. the definition of locally quasiconformally collared in [14, p. 54]. If we could replace "flat" by "collared" in Theorem 1 then we would be able to improve Corollary 4 to give us a generalisation to $\overline{\mathbf{R}}^{n}$ of Gehring's Theorem 4 in [6]. Of course Väisälä's definition of global quasiconformal collaring is the same as our definition of (global) quasiconformal flatness, so that if one wanted to improve Theorem 1 above by replacing "flat" by "collared", one would need to find such a homeomorphism $h$. In fact, an affirmative answer to the following conjecture is precisely what we need.

Conjecture. Let $D$ be a Jordan domain in $\overline{\mathbf{R}}^{n}$. Suppose that for each $x \in \partial D$ there is an open neighbourhood $U$ of $x$ in $\bar{D}$ and an embedding $h_{U}$ of $\bar{U}$ in $\bar{B}^{n}$ so that $h_{U}^{-1}\left(S^{n-1}\right)=\bar{U} \cap \partial D$,

$$
h_{U}(\bar{U})=\left\{r z \in \mathbf{R}^{n} \mid 1 / 2 \leqq r \leqq 1 \text { and } z \in h_{U}(\bar{U} \cap \partial D)\right\}
$$

and $h_{U} \mid U \cap D$ is quasiconformal. Then there is a homeomorphism $g: \partial D \rightarrow S^{n-1}$ so that for each $x \in \partial D$ there is an open neighbourhood $V$ of $x$ in $\bar{D}$ and an extension 
of $g \mid V \cap \partial D$ to a homeomorphism $g_{V}$ of $\bar{V}$ onto

$$
\left\{r z \in \mathbf{R}^{n} \mid 1 / 2 \leqq r \leqq 1 \text { and } z \in g(\bar{V} \cap \partial D)\right\}
$$

so that $g_{V} \mid V \cap D$ is quasiconformal.

This conjecture is true for $n=3$, see [6, Theorem 4].

The topological version of this conjecture (i.e. that obtained by deleting quasiconformality) is trivial, for we may let $g: \partial D \rightarrow S^{n-1}$ by any homeomorphism, and for each $U$, let $f_{U}(r z)=r g h_{U}^{-1}(z)$ for $1 / 2 \leqq r \leqq 1$ and $z \in h_{U}(\bar{U} \cap \partial D)$. Now let $g_{U}=f_{U} h_{U}$.

3. Flattening the boundary of a Jordan domain. In this section we prove Theorem 1 , so suppose that $D$ is a Jordan domain in $\overline{\mathbf{R}}^{n}$ and that $\partial D$ is locally quasiconformally flat in $D$.

Use the notation of the definition of locally quasiconformally flat; we will assume that $a=1 / 3$ in the definition. Since $\partial D$ is compact, we may assume that $\mathscr{U}$ is finite, say $\mathscr{U}=\left\{U_{1}, \ldots, U_{m}\right\}$. Abbreviate $h_{U_{i}}$ to $h_{i}$.

Let $D^{*}$ be the topological space obtained from the disjoint union of $\bar{D}$ and $\partial D \times I[1,3 / 2]$ by identifying $x \in \partial D \subset \bar{D}$ with $(x, 1) \in \partial D \times I[1,3 / 2]$, (see Figure 1). We want to impose a collar on $\partial D$ lying inside $D . D^{*}$ is $D$ together with a collar on $\partial D$. The idea is to push this collar inside $D$.

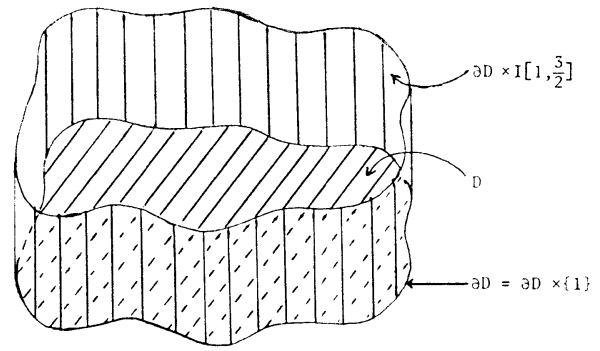

Figure 1

We will define a homeomorphism $e_{m}: \bar{D} \rightarrow D^{*}$ : the extension of $h$ to a neighbourhood of $\partial D$ in $\bar{D}$ required by the definition of quasiconformal flatness will use the product structure of $\partial D \times I(1,3 / 2]$, the neighbourhood in question being $e_{m}^{-1}(\partial D \times I(1,3 / 2])$.

Let $U_{i}^{*}=U_{i} \cup\left(U_{i} \cap \partial D\right) \times I[1,3 / 2]$, and extend $h_{i}$ to

$$
h_{i}^{*}: U_{i}^{*} \rightarrow\left\{r z \in \mathbf{R}^{n} \mid 1 / 3<r \leqq 3 / 2 \text { and } z \in h\left(U_{i} \cap \partial D\right)\right\}
$$

by letting $h_{i}^{*}(x, t)=\operatorname{th}(x)$ when $x \in U_{i} \cap \partial D$ and $t \in I[1,3 / 2]$.

Define the "projections" $p_{i}: U_{i}^{*} \rightarrow U_{i} \cap \partial D$ and $q_{i}: U_{i}^{*} \rightarrow I(1 / 3,3 / 2]$ by $p_{i}(x)=h^{-1}(z)$ and $q_{i}(x)=r$ if $x \in U_{i}$ with $h_{i}(x)=r z$ for $1 / 3<r \leqq 1$ and $z \in h\left(U_{i} \cap \partial D\right)$, and if $(x, t) \in\left(U_{i} \cap \partial D\right) \times I[1,3 / 2]$, let

$$
p_{i}(x, t)=x \quad \text { and } \quad q_{i}(x, t)=t .
$$


Let

$$
\left\{\lambda_{i}^{\prime}: S^{n-1} \rightarrow I[0,1] \mid i=1, \ldots, m\right\}
$$

be a smooth partition of unity subordinate to the cover

$$
\left\{h\left(U_{i} \cap \partial D\right) \mid i=1, \ldots, m\right\}
$$

of $S^{n-1}\left[8\right.$, p. 17], and define $\lambda_{i}: \partial D \rightarrow I[0,1 / 2]$ by

$$
\lambda_{i}(x)=(1 / 2) \lambda_{i}^{\prime} h(x), \quad x \in \partial D .
$$

Define $\mu_{i}: \partial D \rightarrow I[0,1 / 2], i=0, \ldots, m$, by

$$
\mu_{i}(x)=\sum_{j=1}^{i} \lambda_{j}(x), \quad x \in \partial D
$$

Note that $\mu_{0}(\partial D)=0$ and $\mu_{m}(\partial D)=1 / 2$.

Using induction on $k$ and a modification of a construction due to Connelly [3], we now construct embeddings $e_{k}: \bar{D} \rightarrow D^{*}, k=0, \ldots, m$, to satisfy the following properties:

(i) $e_{k}(\bar{D})=\bar{D} \cup\left\{(x, t) \in \partial D \times I[1,3 / 2] \mid t \leqq 1+\mu_{k}(x)\right\}$;

(ii) For every $j=1, \ldots, m$, the embedding $h_{j}^{*} e_{k}$ is quasiconformal on the interior of its domain, i.e. on the interior of $e_{k}^{-1}\left(U_{j}^{*}\right)$.

Induction begins at $k=0$ by letting $e_{0}$ be the inclusion. Then $e_{0}(\bar{D})=\bar{D}$ so (i) is satisfied, and for each $j=1, \ldots, m$, the domain of $h_{j}^{*} e_{0}$ is $U_{j}$ on the interior of which $h_{j}^{*} e_{0}=h_{j}$ is quasiconformal.

Suppose now that $e_{k-1}$ has been constructed so as to satisfy the conditions. Define $e_{k}$ as follows:

(a) if $e_{k-1}(x) \in D^{*}-U_{k}^{*}$ or if $e_{k-1}(x) \in U_{k}$ and $1 / 3<q_{k} e_{k-1}(x) \leqq 1 / 2$, let

$$
e_{k}(x)=e_{k-1}(x) \text {; }
$$

(b) if $e_{k-1}(x) \in U_{k}^{*}$ and $1 / 2 \leqq q_{k} e_{k-1}(x)(\leqq 3 / 2)$, let

$$
e_{k}(x)=h_{k}^{*-1}\left(\left[q_{k} e_{k-1}(x)+\frac{\lambda_{k} p_{k} e_{k-1}(x)\left(2 q_{k} e_{k-1}(x)-1\right)}{1+2 \mu_{k-1} p_{k} e_{k-1}(x)}\right] h p_{k} e_{k-1}(x)\right) .
$$

The effect of part (b) of this definition is as follows: map $x$ to the point $h_{k}^{*} e_{k-1}(x)$ in the "nice" region

$$
\left\{r z \in \mathbf{R}^{n} \mid 1 / 2 \leqq r \leqq 3 / 2 \text { and } z \in h\left(U_{k} \cap \partial D\right)\right\},
$$

apply a radial diffeomorphism to the image, this radial diffeomorphism stretching, for each $z \in h\left(U_{k} \cap \partial D\right)$, the radial line

onto the radial line

$$
\left\{r z \in \mathbf{R}^{n} \mid 1 / 2 \leqq r \leqq 1+\mu_{k-1} h^{-1}(z)\right\}
$$

$$
\left\{r z \in \mathbf{R}^{n} \mid 1 / 2 \leqq r \leqq 1+\mu_{k} h^{-1}(z)\right\},
$$

and then apply $h_{k}^{*-1}$. (See Figure 2 .) 
Well-definition of $e_{k}$ : if $e_{k-1}(x) \in U_{k}^{*}$ and $1 / 2 \leqq q_{k} e_{k-1}(x)$, then by inductive assumption (i),

and since also

$$
1 / 2 \leqq q_{k} e_{k-1}(x) \leqq 1+\mu_{k-1} p_{k} e_{k-1}(x),
$$

we have that

$$
0 \leqq \lambda_{k} p_{k} e_{k-1}(x) \leqq 1 / 2-\mu_{k-1} p_{k} e_{k-1}(x) \text {, }
$$

(*) $\quad \frac{1}{2} \leqq q_{k} e_{k-1}(x)+\frac{\lambda_{k} p_{k} e_{k-1}(x)\left(2 q_{k} e_{k-1}(x)-1\right)}{1+2 \mu_{k-1} p_{k} e_{k-1}(x)} \leqq 1+\mu_{k} p_{k} e_{k-1}(x)$.

Since $1+\mu_{k} p_{k} e_{k-1}(x) \leqq 3 / 2$, we see that

$$
\left[q_{k} e_{k-1}(x)+\frac{\lambda_{k} p_{k} e_{k-1}(x)\left(2 q_{k} e_{k-1}(x)-1\right)}{1+2 \mu_{k-1} p_{k} e_{k-1}(x)}\right] h p_{k} e_{k-1}(x)
$$

is in the image of the embedding $h_{k}^{*}$, so (b) makes sense.
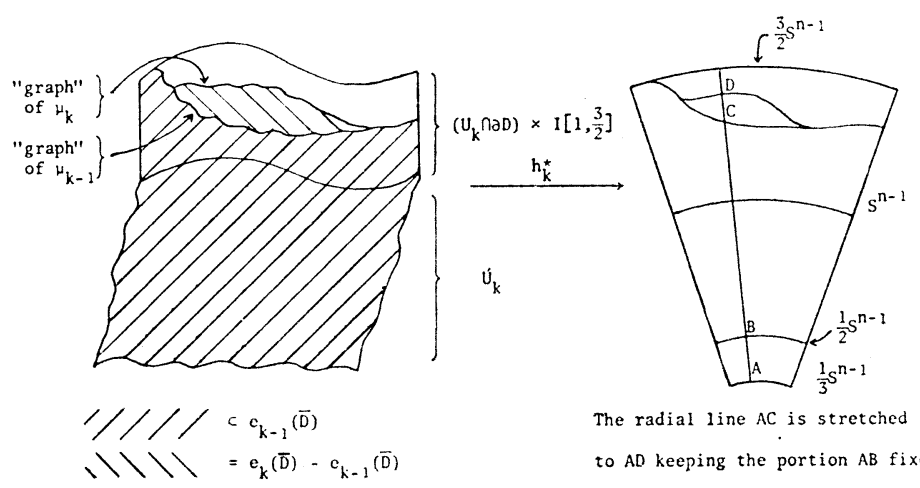

The radial line $A C$ is stretched

to $A D$ keeping the portion $A B$ fixed.

Figure 2

If $e_{k-1}(x) \in U_{k}$ and $q_{k} e_{k-1}(x)=1 / 2$, then the left inequality of (*) becomes an equality, and definition (b) of $e_{k}(x)$ reduces to

$$
\begin{aligned}
& h_{k}^{*-1}\left((1 / 2) h p_{k} e_{k-1}(x)\right) \\
= & h_{k}^{-1}\left(q_{k} e_{k-1}(x) \cdot h p_{k} e_{k-1}(x)\right) \\
= & e_{k-1}(x),
\end{aligned}
$$

so definitions (a) and (b) are compatible.

If $e_{k-1}(x) \in U_{k}^{*}$ and $1 / 2 \leqq q_{k} e_{k-1}(x)$, then, provided $p_{k} e_{k-1}(x)$ is sufficiently close to the frontier of $U_{k} \cap \partial D$ in $\partial D$, we have $\lambda_{k} p_{k} e_{k-1}(x)=0$; this being so because the partition of unity $\left\{\lambda_{i}^{\prime} \mid i=1, \ldots, m\right\}$ is subordinate to the cover $\left\{h\left(U_{i} \cap \partial D\right) \mid i=1, \ldots, m\right\}$. In this case, definition (b) of $e_{k}(x)$ again reduces to $e_{k-1}(x)$. 
Thus $e_{k}$ is continuous. Furthermore, $e_{k}$ is clearly injective, so, being a continuous injective function from a compact space to a Hausdorff space, it is an embedding.

We must verify that $e_{k}$ satisfies the properties (i) and (ii).

(i) We need only verify the truth of the equality when restricted to $U_{k}^{*}$, since only here do $e_{k-1}$ and $e_{k}$, and $\mu_{k-1}$ and $\mu_{k}$ differ. By inductive hypothesis (i), $q_{k} e_{k-1}(x)$ can be as large as $1+\mu_{k-1} p_{k} e_{k-1}(x)$, in which case the right inequality of (*) becomes an equality. Thus the frontier of $e_{k-1}(\bar{D})$ has been pushed, in $U_{k}^{*}$, out as far as $\left\{(x, t) \in \partial D \times I[1,3 / 2] \mid t=1+\mu_{k}(x)\right\}$ to construct $e_{k}(\bar{D})$.

Clearly $e_{k}(\bar{D})$ also includes everything out to this frontier, so property (i) is satisfied.

(ii) Again we need only verify this statement inside $e_{k-1}^{-1}\left(U_{k}^{*}\right)$, since $e_{k}$ agrees with $e_{k-1}$ off $e_{k-1}^{-1}\left(U_{k}^{*}\right)$. Let $j$ be an integer between 1 and $m$. Referring to definition (b) of $e_{k}$, we see that $h_{j}^{*} e_{k}=\left(h_{j}^{*} h_{k}^{*-1}\right) \circ$ (stretching diffeomorphism) $\circ\left(h_{k}^{*} e_{k-1}\right)$, a composition of three maps. By inductive assumption (ii), $h_{k}^{*} e_{k-1}$ is quasiconformal; any diffeomorphism is quasiconformal provided mild conditions are satisfied (see $[14$, p. 48$]$; these conditions are satisfied in our situation); $h_{j}$ and $h_{k}^{-1}$ are quasiconformal, hence so is their composition $h_{j} h_{k}^{-1}$ where defined. On $h_{k}^{*}\left[\left(U_{k}^{*} \cap U_{j}^{*}\right)-\right.$ $\left.-\left(U_{k} \cap U_{j}\right)\right], h_{j}^{*} h_{k}^{*-1}$ is the identity, which is quasiconformal. Thus $h_{j}^{*} h_{k}^{*-1}$ is quasiconformal, and we have expressed $h_{j}^{*} e_{k}$ as a composition of three quasiconformal mappings, so $h_{j}^{*} e_{k}$ is quasiconformal.

This completes our inductive construction of the embeddings $e_{k}$.

As already noted, $\mu_{m}(\partial D)=1 / 2$, so property (i) of $e_{m}$ tells us that $e_{m}(\bar{D})=D^{*}$, i.e. $e_{m}$ is a homeomorphism. Let

$$
U=e_{m}^{-1}(\partial D \times I[1,3 / 2]) .
$$

Then $U$ is a neighbourhood of $\partial D$ in $\bar{D}$. Define

by

$$
H: U \rightarrow[1 / 2,1]
$$

$$
H(x)=\left(q e_{m}(x)-1 / 2\right) h p e_{m}(x),
$$

where $p: \partial D \times I[1,3 / 2] \rightarrow \partial D$ and $q: \partial D \times I[1,3 / 2] \rightarrow I[1,3 / 2]$ are the standard projections.

(I) $\quad H$ is a homeomorphism with the above range. In fact, $H$ is the composition of three homeomorphisms: $e_{m}$, taking $U$ onto $\partial D \times I[1,3 / 2]$; the obvious radial extension of $h$ to a homeomorphism from $\partial D \times I[1,3 / 2]$ onto the annulus [1,3/2] in $\mathbf{R}^{n}$ (cf. the extension of $h_{i}$ to $h_{i}^{*}$ ); and a radial shrinking of [1,3/2] to [1/2,1]. (II) $H$ extends $h$. If $x \in \partial D$, then one can easily see from definition (b) of $e_{k}$ that $e_{k}(x)=\left(x, 1+\mu_{k}(x)\right)$, so that $e_{m}(x)=(x, 3 / 2)$, and hence

$$
p e_{m}(x)=x \text { and } q e_{m}(x)=3 / 2 .
$$

Thus $H(x)=h(x)$ as required. 
(III) $H$ is quasiconformal on $U \cap D$. This follows from inductive assumption (ii). For any $j=1, \ldots, m$, by (ii), $h_{j}^{*} e_{m}$ is quasiconformal on $U \cap D \cap e_{m}^{-1}\left(U_{j}^{*}\right)$. Since on such a set $H$ is the composition $h_{j}^{*} e_{m}$ followed by the shrinking, $H$ is quasiconformal on $U \cap D \cap e_{m}^{-1}\left(U_{j}^{*}\right)$. Thus $H$ is quasiconformal since

$$
\left\{U \cap D \cap e_{m}^{-1}\left(U_{j}^{*}\right) \mid j=1, \ldots, m\right\}
$$

is a finite open cover of $U \cap D$.

By (I), (II) and (III), $\partial D$ is quasiconformally flat in $D$ as required.

Remark. We can assume that $\lambda_{1}^{\prime}$ is the identity on a given compact subset $K$ of $h\left(U_{1} \cap \partial D\right)$. In this case, the mapping $H$ will agree with $h_{1}$ on $V_{1}=U \cap$ $\cap p_{1}^{-1} h^{-1}(K)$, so that we can extend the collar $h_{1} \mid V_{1}$ to the whole of $\partial D$.

4. The boundary of a non-Jordan domain. Suppose $D$ is some domain in $\overline{\mathbf{R}}^{n}$ and $\partial D$ is a topological $(n-1)$-manifold without boundary. Provided we can find a model for $\partial D$ which is nicely embedded in $\overline{\mathbf{R}}^{n}$, the techniques of $\S 3$ generalise to such a $D$. The main criterion for "niceness" of the model, say $M$, is that there is an embedding of $M \times[0,1)$ in $\overline{\mathbf{R}}^{n}$ whose restriction to $M \times\{0\}$ is the projection on $M$. The $[0,1)$ factor of this embedding plays the same role in this case as did the radial structure of $\overline{\mathbf{R}}^{n}$ in the previous case. As an example, the 2-torus $T^{2}=S^{1} \times S^{1}$ has a nice embedding in $\mathbf{R}^{3}$ obtained by revolving the circle

$$
(x-2)^{2}+z^{2}=1, \quad y=0
$$

around the $z$-axis. Given a domain $D$ in $\overline{\mathbf{R}}^{3}$ whose boundary $\partial D$ is homeomorphic to $T^{2}$, we can speak of local quasiconformal flatness of $\partial D$ in $D$ and the techniques used to prove Theorem 1 carry over to this case to prove that if $\partial D$ is locally quasiconformally flat in $D$ then $\partial D$ is quasiconformally flat in $D$.

In certain cases where $\partial D$ is not an $(n-1)$-manifold we can still use the above techniques to go from local flatness to global flatness, an example being provided by Theorem 2 .

Proof of Theorem 2. We slit $\overline{\mathbf{R}}^{n}$ along $X$ to obtain a space $Y$ and a subset $Z$ of $Y$ corresponding to the slit $X . Z$ is locally quasiconformally flat in $Y$, so by Theorem 1 is quasiconformally flat in $Y$. This flatness structure gives us the desired extension of $h$.

Precisely, let

$$
Y=\left[\overline{\mathbf{R}}^{n}-X\right] \cup\left([X \times\{-1,1\}] \mid\left[(x,-1) \sim(x, 1) \text { for } x \in h^{-1}\left(S^{n-2}\right)\right]\right) .
$$

Define $\pi: Y \rightarrow \overline{\mathbf{R}}^{n}$ by $\pi(y)=y$ if $y \in \overline{\mathbf{R}}^{n}-X$ and $\pi(x, \pm 1)=x$ if $x \in X$. The function $\pi$, the identity on $\overline{\mathbf{R}}^{n}-X$, closes up the slit.

By Möbius transformations, $\overline{\mathbf{R}}^{n}-\bar{B}^{n}$ and $\overline{\mathbf{R}}^{n}-\bar{B}^{n-1}$ are, conformally, open half space and slit space respectively. Let $\varrho: \overline{\mathbf{R}}^{n}-B^{n} \rightarrow \overline{\mathbf{R}}^{n}$ be such that $\varrho$ folds the half space $\overline{\mathbf{R}}^{n}-\bar{B}^{n}$ quasiconformally onto $\overline{\mathbf{R}}^{n}-\bar{B}^{n-1}$. Then $\overline{\mathbf{R}}^{n}-B^{n}$ is essentially $\overline{\mathbf{R}}^{n}$ slit along $B^{n-1}$ and $\varrho$ closes up this slit in the same way as $\pi$ closes the slit $X$. 
Let $Z=\pi^{-1}(X)$ with the quotient topology inherited from $X \times\{-1,1\}$, and choose a homeomorphism $g: Z \rightarrow S^{n-1}$ so that the following diagram commutes:

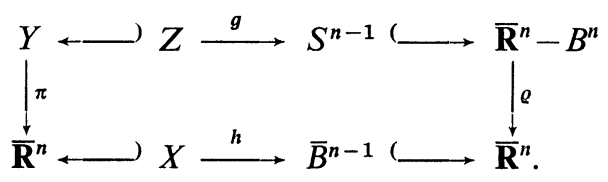

In fact, $h$ has only two such liftings: either one will do.

For each $U \in \mathscr{U}$, we can extend $g \mid \pi^{-1}(U) \cap Z$ uniquely to an injective function

$$
g_{U}: \pi^{-1}(U) \rightarrow \overline{\mathbf{R}}^{n}-B^{n}
$$

which lifts $h_{U}$, making the following diagram commute:

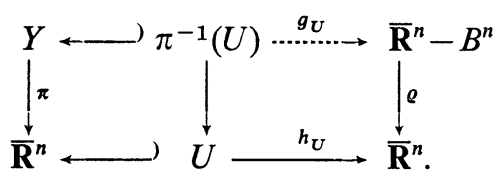

Topologise $Y$ by declaring the union of the following two families to be a basis:

$$
\begin{gathered}
\left\{V \subset \overline{\mathbf{R}}^{n}-X \mid V \text { is open in } \overline{\mathbf{R}}^{n}\right\} ; \\
\left\{g_{U}^{-1}(V) \mid U \in \mathscr{U} \text { and } V \text { is open in } \overline{\mathbf{R}}^{n}-B^{n}\right\} .
\end{gathered}
$$

The effect of this is to slit any open subset of $\overline{\mathbf{R}}^{n}$ along $X$ into two open sets, one on each side of the slit. Under this topology, $g$ is a homeomorphism and each $g_{U}$ is an embedding. Since the topology on the subspace $Y-Z=\overline{\mathbf{R}}^{n}-X$ of $Y$ is the usual topology, one may speak of quasiconformality of the functions $g_{U} \mid(Y-Z) \cap \pi^{-1}(U)$. In fact these functions are quasiconformal since they agree with $\varrho^{-1} h_{U} \mid\left(\overline{\mathbf{R}}^{n}-X\right) \cap U$. Thus $\left\{g_{U} \mid U \in \mathscr{U}\right\}$ provides a local quasiconformal flatness structure for $Z$ in $Y$. The techniques of Theorem 1 can now be applied to extend $g$ to an embedding $\bar{g}: N \rightarrow \overline{\mathbf{R}}^{n}-B^{n}$, where $N$ is a neighbourhood of $Z$ in $Y$ (and hence $g(N)$ is a neighbourhood of $S^{n-1}$ in $\left.\overline{\mathbf{R}}^{n}-B^{n}\right)$, so that $\bar{g} \mid(Y-Z) \cap N$ is quasiconformal.

Define $\bar{h}: \pi(N) \rightarrow \overline{\mathbf{R}}^{n}$ by $\bar{h}(x)=\varrho \bar{g} \pi^{-1}(x)$. Then $\bar{h}$ extends $h$ and is an embedding of the neighbourhood $\pi(N)$ of $X$ in $\overline{\mathbf{R}}^{n}$. Moreover $\bar{h}$ is quasiconformal on $\pi(N)-X$ and hence on $\pi(N)$, since $\bar{B}^{n-1}$ is of finite $(n-1)$-measure, [14].

5. Inside a Jordan domain. In this section we give our proof of Theorem 3. Brown's proof in [2] consists essentially of two distinct steps, (cf. [12, p. 48]).

Step I. Let $N=[1 / 2,1]$, a neighbourhood of $S^{n-1}$ in $\bar{B}^{n}$, and suppose $e: N \rightarrow \overline{\mathbf{R}}^{n}$ is an embedding. Let $C$ denote the component of $\overline{\mathbf{R}}^{n}-e\left(S^{n-1}\right)$ for which $e(N) \subset \bar{C}$. Then there is an embedding $f: \bar{B}^{n} \rightarrow \overline{\mathbf{R}}^{n}$ so that $f\left(B^{n}\right) \cup$ $\cup e((1 / 2,1))=C$. (See Figure 3.) 
Step II. Given embeddings $e$ and $f$ as in Step I, there is an embedding $\hat{e}: \bar{B}^{n} \rightarrow \overline{\mathbf{R}}^{n}$ for which $\hat{e}(x)=e(x)$ whenever $x \in S^{n-1}$, and $\hat{e}\left(\bar{B}^{n}\right)=\bar{C}$.

Step I involves the use of non-quasiconformal embeddings even when $e$ is quasiconformal. However, in the proof of this step in Lemma 7 below, we see that the embedding $f$ is quasiconformal when $e$ is quasiconformal.

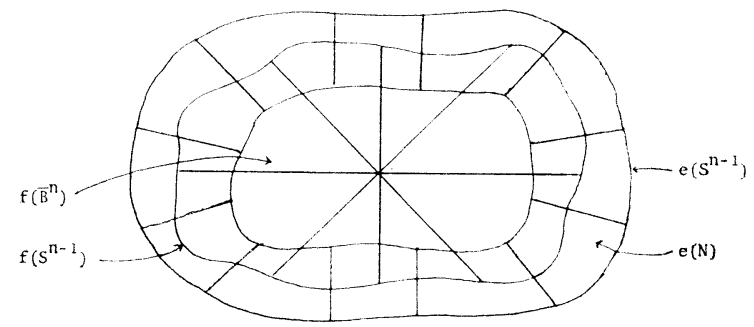

Figure 3

It is at Step II that the non-quasiconformality referred to by Väisälä arises, since Brown's proof of this step involves an interplay between the radial structures of $e(N)$ and of $f\left(\bar{B}^{n}\right)$, the embedding $\hat{e}$ being the limit of a sequence of embeddings

$$
e_{k}: S^{n-1} \times[0,1] \rightarrow \overline{\mathbf{R}}^{n}
$$

where $e_{k}(x, 0)=e(x)$ for $x \in S^{n-1}: \lim _{k \rightarrow \infty} e_{k}$ is an embedding off $S^{n-1} \times\{1\}$ and sends $S^{n-1} \times\{1\}$ to a single point. Since $S^{n-1} \times[0,1] / S^{n-1} \times\{1\}$ is naturally homeomorphic to $\bar{B}^{n}$, we may think of $\lim _{k \rightarrow \infty} e_{k}$ as being an embedding of $\bar{B}^{n}$ in $\overline{\mathbf{R}}^{n}$. Now each $e_{k}$ may be assumed quasiconformal if $e$ and $f$ are (to give this meaning, we may identify $S^{n-1} \times[0,1]$ with $[1 / 2,1]$ in a natural way) but the limit of the $e_{k}$ 's need not be: each $e_{k}$ is, say $K_{k}$-quasiconformal, but $\lim _{k \rightarrow \infty} K_{k}$ may be infinite (contrast 37.3 on p. 125 of [14]).

Below we split Brown's Step II into two parts; see Lemmas 8 and 9. The first part is essentially Brown's construction of $e_{1}$ and the second part is an adaptation of ideas in [5]. We will find that if $e$ and $f$ are quasiconformal then so is $\hat{e}$.

Lemma 7. Let $e:[1 / 2,1] \rightarrow \overline{\mathbf{R}}^{n}$ be an embedding and denote by $C$ the component of $\overline{\mathbf{R}}^{n}-e\left(S^{n-1}\right)$ for which $e([1 / 2,1)) \subset C$. Then there is an embedding $f: \bar{B}^{n} \rightarrow \overline{\mathbf{R}}^{n}$ satisfying

$$
f\left(\bar{B}^{n}\right) \cup e((1 / 2,1))=C .
$$

If $e$ is quasiconformal, then so is $f$.

Proof. Write $N=[1 / 2,1]$. By performing a (conformal) transformation if necessary, we may assume that $\infty \in e((1 / 2,1)), \partial e(N) \subset B^{n}$ and $0 \in C_{2}$, where $C_{2}$ denotes the bounded component of $\overline{\mathbf{R}}^{n}-e\left((1 / 2) S^{n-1}\right)$. Let $C_{1}$ denote the bounded component of $\overline{\mathbf{R}}^{n}-e\left(S^{n-1}\right)$. Then $C_{1} \cup C_{2} \subset B^{n}$. (See Figure 4.) 


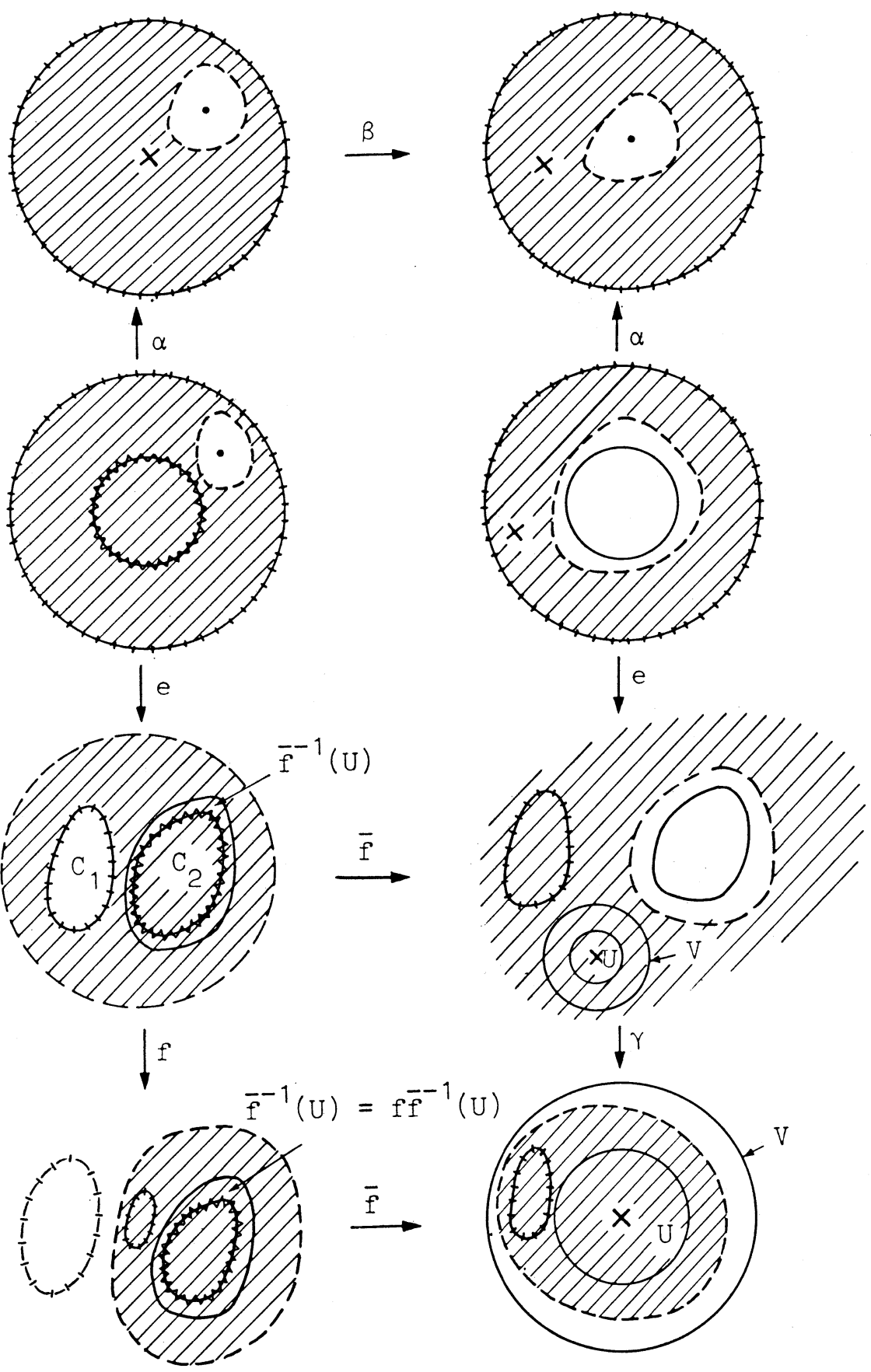

Figure 4 
Let $\alpha:[1 / 2,1] \rightarrow \bar{B}^{n}$ be the radial stretching given by $\alpha(t x)=(2 t-1) x$ for $1 / 2 \leqq t \leqq 1,\|x\|=1$. Then $\alpha e^{-1}$ has a natural continuous extension over $C_{2}$ which sends $C_{2}$ to 0 . Next, let $\beta: \overline{\mathbf{R}}^{n} \rightarrow \overline{\mathbf{R}}^{n}$ be a quasiconformal mapping which is the identity on $\overline{\mathbf{R}}^{n}-B^{n}$ so that $\beta\left(\alpha e^{-1}(\infty)\right)=0$.

Define $\bar{f}: \mathbf{R}^{n} \rightarrow \overline{\mathbf{R}}^{n}$ by

$$
f(x)=\left\{\begin{array}{lll}
e \alpha^{-1} \beta \alpha e^{-1}(x) & \text { for } & x \notin C_{1}, \\
x & \text { for } & x \in C_{1} .
\end{array}\right.
$$

If $x \in \partial C_{1}$ then $\alpha e^{-1}(x) \in S^{n-1}$ where $\beta$ is the identity, so for such $x, f(x)=x$. Thus $\bar{f}$ is continuous. Although $\bar{f}$ is an embedding only on $\mathbf{R}^{n}-\bar{C}_{2}$, and $\bar{f}$ is not quasiconformal on $\mathbf{R}^{n}-\bar{C}_{2}$, when $e$ is quasiconformal $\bar{f}$ is quasiconformal on each compact subset of $\mathbf{R}^{n}-\bar{C}_{2}$ which is all we will need.

Let $p=\bar{f}\left(\bar{C}_{2}\right)$ and let $U \subset V$ and $W$ be balls with $U$ and $V$ centred at $p, V$ of twice the radius of $U$, so that $\bar{V} \subset e((1 / 2,1)), W$ centred at 0 so that $\bar{W} \subset C_{2}$. Let $\gamma: \overline{\mathbf{R}}^{n} \rightarrow \overline{\mathbf{R}}^{n}$ be a quasiconformal mapping which maps the annulus $\overline{\mathbf{R}}^{n}-(U \cup W)$ onto the annulus $\bar{V}-U$ leaving points of $\bar{U} \cup\{0\}$ fixed.

Now define the required function $f$ by

$$
f(x)=\left\{\begin{array}{lll}
\bar{f}^{-1} \gamma \vec{f}(x) & \text { if } & x \in e((1 / 2,1]) \cup C_{1}, \\
x & \text { if } & x \in \bar{f}^{-1}(U) .
\end{array}\right.
$$

Then $f$ is an embedding satisfying $f\left(\bar{B}^{n}\right) \cup e((1 / 2,1))=C$. Moreover, if $e$ is quasiconformal on $(1 / 2,1)$ then $f$ is quasiconformal on $B^{n}$.

Lemma 8. Let $e$ and $f$ be as in Lemma 7. Then there exist real numbers $a, b, c$ and an embedding $\hat{f}: \bar{B}^{n} \rightarrow \overline{\mathbf{R}}^{n}$ satisfying:

(i) $0<a<b<1,1 / 2<c<1$;

(ii) $\hat{f}([b, 1]) \subset e((c, 1))$;

(iii) $\hat{f}([a]) \subset e((1 / 2, c))$.

(iv) For every $x \in S^{n-1}, \hat{f}(x)=f(x)$.

Further, if $e$ is quasiconformal then so is $\hat{f}$.

Proof. Let $C$ and $C_{2}$ be as in Lemma 7. We may assume that $\bar{C}$ is bounded and that $f(0) \in C_{2}$.

Now $f\left(S^{n-1}\right) \subset e((1 / 2,1))$, so there exists $b$ with $b<1$ such that $f([b, 1]) \subset$ $\subset e((1 / 2,1))$. Thus $e([1 / 2]) \subset f((0, b))$, so there exists $c$ with $1 / 2<c$ such that $e([1 / 2, c]) \subset f((0, b))$. Since $f(0) \in C_{2}$, there exists $a$ with $0<a$ such that $f([0, a]) \subset C_{2}$. See Figure 5 .

Define $s:[0,1] \rightarrow[0, c]$ to be that homeomorphism which fixes $[0,1 / 2]$ and radially shrinks $[1 / 2,1]$ to $[1 / 2, c]$, and define $\sigma:[0,1] \rightarrow[0,1]$ to be that homeomorphism which stretches $[0, a]$ radially to $[0, b]$ and shrinks $[a, 1]$ radially to $[b, 1]$. Define $S, \Sigma: \bar{C} \rightarrow \bar{C}$ by

$$
S(x)= \begin{cases}e s e^{-1}(x) & \text { if } x \in e([1 / 2,1]), \\ x & \text { otherwise }\end{cases}
$$


and

$$
\Sigma(x)= \begin{cases}f \sigma f^{-1}(x) & \text { if } x \in f\left(\bar{B}^{n}\right), \\ x & \text { otherwise. }\end{cases}
$$

Clearly $S$ and $\Sigma$ satisfy the quasiconformality conditions. Define the required embedding $\hat{f}$ (cf. Figure 6 ) by

$$
\hat{f}(x)= \begin{cases}f(x) & \text { if } f(x) \in e([c, 1]), \\ S \Sigma S^{-1} f(x) & \text { otherwise. }\end{cases}
$$

Then $\hat{f}$ satisfies the required conditions.

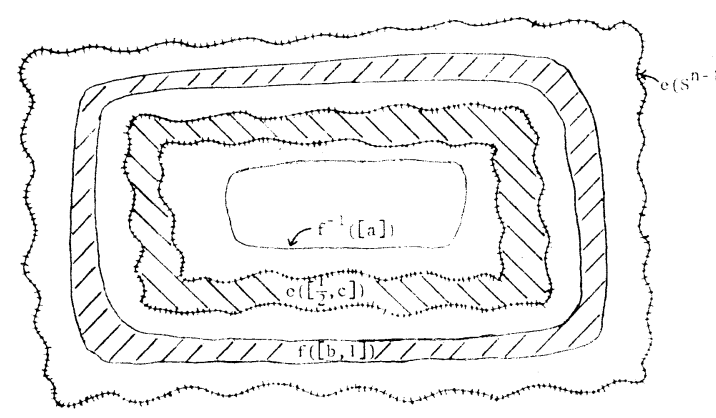

Figure 5
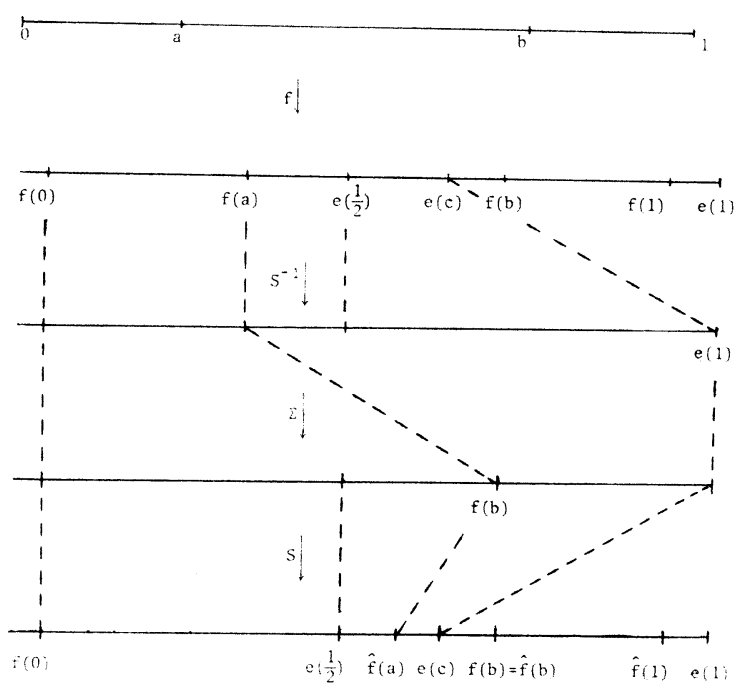

Figure 6

Lemma 9. Let $e:[1 / 2,1] \rightarrow \overline{\mathbf{R}}^{n}$ be an embedding and $a, b$ and $c$ be real numbers satisfying:

(i) $0<a<b<1,1 / 2<c<1$;

(ii) $[b, 1] \subset e((c, 1))$;

(iii) $[a] \subset e((1 / 2, c))$. 
Then there is an embedding $\hat{e}: \bar{B}^{n} \rightarrow \overline{\mathbf{R}}^{n}$ such that $\hat{e}(x)=e(x)$ if $\|x\| \geqq c$. Moreover, if $e$ is quasiconformal then so is $\hat{e}$.

Proof. Choose $d \in I(1 / 2, c)$ so that $[a] \subset e((1 / 2, d))$. the following:

Let $x, \lambda, \mu: \overline{\mathbf{R}}^{n} \rightarrow \overline{\mathbf{R}}^{n}$ be the natural radial homeomorphisms doing

$$
\begin{array}{rlrl}
\varkappa([0,1 / 2]) & =[0, c] ; & \varkappa([1 / 2, c]) & =[c, 1] ; \\
\lambda([0, b]) & =[0, a] ; & \lambda([b, 1])=[a, 1], \quad \lambda([1, \infty])=[1, \infty] ; \\
\mu([0, d]) & =[0, d] ; & \mu([d, 1])=[d, c] .
\end{array}
$$

Define the embedding $\bar{e}:[1 / 2,1] \rightarrow \overline{\mathbf{R}}^{n}$ (see Figure 7) by

$$
\bar{e}(x)= \begin{cases}e(x) & \text { if }\|x\| \geqq c, \\ e \mu e^{-1} \lambda e x(x) & \text { if }\|x\| \leqq c \text { and } \lambda \operatorname{ex}(x) \in e([d, 1]), \\ \lambda e x(x) & \text { if } \lambda \operatorname{ex}(x) \in e([1 / 2, d]) \cup[0, a] .\end{cases}
$$

Well-definition of $\bar{e}$ :

$(\alpha)$ If $1 / 2 \leqq\|x\| \leqq c$ then $x(x) \in[c, 1]$ which is in the domain of $e$, so $e x(x)$ is defined and hence so is $\lambda e x(x)$. If, further $\lambda \operatorname{ex}(x) \in e([d, 1])$, then

so

$$
e^{-1} \lambda \operatorname{ex}(x) \in[d, 1]
$$

$$
\mu e^{-1} \lambda e x(x) \in[d, c]
$$

which also lies in the domain of $e$, so $e \mu e^{-1} \lambda e x(x)$ is defined.

( $\beta)$ If $\lambda \operatorname{ex}(x) \in e\left(d S^{n-1}\right)$, then $e^{-1} \lambda e x(x) \in d S^{n-1}$, and so

$$
e \mu e^{-1} \lambda e x(x)=e e^{-1} \lambda e x(x)=\lambda e x(x) .
$$

Thus $\bar{e}$ is well-defined on $x^{-1} e^{-1} \lambda^{-1} e\left(d S^{n-1}\right)$.

(y) If $\|x\|=c, x(x) \in S^{n-1}$, so by (ii), $\quad e x(x) \notin B^{n}$, and hence $\lambda \operatorname{ex}(x)=e x(x)$. Thus

$$
e \mu e^{-1} \lambda e \varkappa(x)=e \mu e^{-1} e \varkappa(x)=e \mu \varkappa(x)=e(x) .
$$

Thus $\bar{e}$ is well-defined on $c S^{n-1}$.

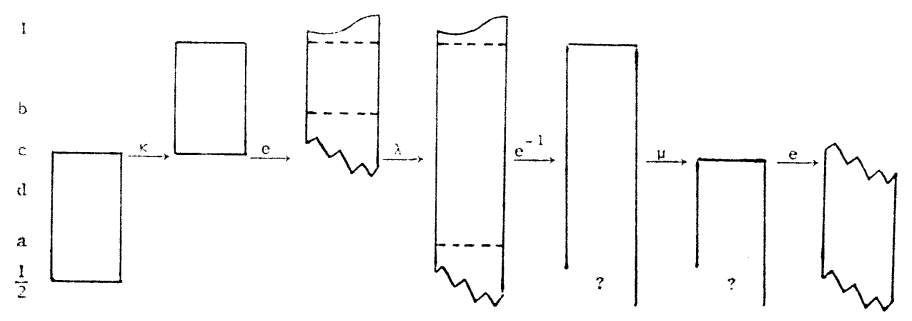

Figure 7 
Combining $(\alpha),(\beta)$, and $(\gamma)$, we see that $\bar{e}$ is well-defined.

It is easily verified that $\bar{e}$ is continuous; that it is an embedding follows from the Jordan-Brouwer separation theorem and invariance of domain (18.6 and 18.9 of [9]).

Note that $\bar{e}$ enjoys the following properties:

(a) if $x \in[c, 1]$ then $\bar{e}(x)=e(x)$;

(b) if $\|x\|=1 / 2$ then $\bar{e}(x)=(a / b) \bar{e}(2 c x)$.

Basically what we have done is to alter $e$ in $[1 / 2, c]$ to get $\bar{e}$, which essentially repeats on [1/2] the action of $e$ on [c]. Following [5] we exploit this repetitive feature of $\bar{e}$ to allow us to extend $\bar{e}$ to $\bar{B}^{n}$.

For $x \in[1 / 2,1]$, we set $\hat{e}(x)=\bar{e}(x)$, and let $\hat{e}(0)=0$. Suppose $x \in(0,1 / 2]$. Then for some non-negative integer $k,(2 c)^{k} x \in[1 / 2, c]$. Let

$$
\hat{e}(x)=(a / b)^{-k} \bar{e}\left((2 c)^{k} x\right) .
$$

By property (b) of $\bar{e}, \hat{e}$ is well-defined, hence continuous except possibly at 0 . Continuity at 0 follows from the fact that $a<b$ so $(a / b)^{-k} \rightarrow 0$ as $k \rightarrow \infty$.

That $\hat{e}$ is an embedding follows as for $\bar{e}$ above.

It remains to verify the quasiconformality of $\hat{e}$ when $e$ is quasiconformal. The embedding $\bar{e}$ is quasiconformal since each of the auxiliary functions $x, \lambda, \mu$ is quasiconformal; say $\bar{e}$ is $K$-quasiconformal. On each open annulus $\left((1 / 2)(2 c)^{-k}\right.$, $\left.c(2 c)^{-k}\right), \hat{e}$ is obtained from $\bar{e}$ by composing with two multiplications, which are conformal. Thus $\hat{e}$ is $K$-quasiconformal on each such annulus. The exceptional set,

$$
\bigcup_{k=0}^{\infty} \frac{1}{2}(2 c)^{-k} S^{n-1} \cup\{0\}
$$

is of finite $(n-1)$-Lebesgue measure and hence is removable [14]. Thus $\hat{e}$ is $K$ quasiconformal on $B^{n}$.

Remark. As in [5], one could replace $\mathbf{R}^{n}$ and $r \bar{B}^{n}$ (which are cones over $S^{n-1}$ ) by cones over more general spaces, although one might want to impose such conditions as compactness, local connectedness and metrisability dictated to us as in [5] by Proposition 3 of [5] and 1.7 of [13].

Proof of Theorem 3. Since $N$ must contain some annulus, we may assume $N=[1 / 2,1]$. Let $a, b, c$ and $\hat{f}: \bar{B}^{n} \rightarrow \overline{\mathbf{R}}^{n}$ be as in Lemma 8 . Let $\tilde{c}:[1 / 2,1] \rightarrow[1 / 2, c]$ denote the natural (quasiconformal) homeomorphism fixing [1/2], and consider the embedding $\varepsilon:[1 / 2,1] \rightarrow \overline{\mathbf{R}}^{n}$ defined by $\varepsilon(x)=(1 / a) \hat{f}^{-1} e \tilde{c}(x)$. By Lemma 8 , taking $f=$ inclusion, there exist real numbers $\alpha, \beta$ and $\gamma$ and an embedding $\hat{\varphi}: \bar{B}^{n} \rightarrow \overline{\mathbf{R}}^{n}$ satisfying:

$$
0<\alpha<\beta<1, \quad 1 / 2<\gamma<1
$$

$$
\hat{\varphi}([\beta, 1]) \subset \varepsilon((\gamma, 1)) ; \quad \hat{\varphi}([\alpha]) \subset \varepsilon((1 / 2, \gamma)) ; \quad \hat{\varphi}(x)=x \text { for } x \in S^{n-1} \text {. }
$$


Define $\zeta:[1 / 2,1] \rightarrow \overline{\mathbf{R}}^{n}$ by

Then:

$$
\zeta(x)=\left\{\begin{array}{lll}
\hat{\varphi}^{-1} \varepsilon(x) & \text { if } & \|\varepsilon(x)\| \leqq 1, \\
\varepsilon(x) & \text { if } & \|\varepsilon(x)\| \geqq 1 .
\end{array}\right.
$$

(i) $0<\alpha<\beta<1,1 / 2<\gamma<1$;

(ii) $[\beta, 1] \subset \zeta((\gamma, 1))$;

(iii) $[a] \subset \zeta((1 / 2, \gamma))$.

Hence by Lemma 9, there exists an embedding $\hat{\zeta}: \bar{B}^{n} \rightarrow \overline{\mathbf{R}}^{n}$ such that $\hat{\zeta}(x)=\zeta(x)$

if $\|x\| \geqq \gamma$. Further, $\hat{\zeta}$ is quasiconformal if $e$ is.

Now define the required embedding $\hat{e}: \bar{B}^{n} \rightarrow \overline{\mathbf{R}}^{n}$ by

$$
\hat{e}(x)= \begin{cases}\hat{f}\left(a \hat{\zeta} \tilde{c}^{-1}(x)\right) & \text { if }\|x\| \geqq c, \\ e(x) & \text { if }\|x\| \geqq c .\end{cases}
$$

Note that if $\|x\|=c$, then $\left\|\tilde{c}^{-1}(x)\right\|=1$, so that $\hat{\zeta} \tilde{c}^{-1}(x)=\zeta \tilde{c}^{-1}(x)$. In this case,

$$
\varepsilon \tilde{c}^{-1}(x)=\frac{1}{a} \hat{f}^{-1} e(x)
$$

which, by (iii) of Lemma 8 , lies outside $B^{n}$. Thus

so that

$$
\hat{\zeta} \tilde{c}^{-1}(x)=\varepsilon \tilde{c}^{-1}(x)=\frac{1}{a} \hat{f}^{-1} e(x),
$$

and $\hat{e}$ is well-defined.

$$
\hat{f}\left(a \zeta \tilde{c}^{-1}(x)\right)=e(x)
$$

Quasiconformality of $\hat{e}$ in the case where $e$ is quasiconformal is readily verified.

Remark. As in [4] and [5], the constructions in Lemmas 7, 8 and 9 are canonical in the sense that if we furnish the various function spaces with the compact-open topologies, then the real numbers and embeddings constructed depend continuously on the given data. For example, Lemma 7 constructs a continuous function (cf. [4])

$$
\mathscr{E}\left([1 / 2,1], \overline{\mathbf{R}}^{n}\right) \rightarrow \mathscr{E}\left(\bar{B}^{n}, \overline{\mathbf{R}}^{n}\right), \quad e \mapsto f ;
$$

here $\mathscr{E}(X, Y)$ denotes the space of embeddings of $X$ in $Y$ with the compactopen topology. Similar interpretations apply to Lemmas 8 and 9. Combining all of these gives us a canonical (quasiconformal) Schoenflies theorem in the sense of [4]. More precisely, we have the following

Canonical quasiconformal Schoenflies theorem. Let $N$ be a neighbourhood of $S^{n-1}$ in $\bar{B}^{n}$. Then there is a continuous function

$$
\varepsilon: \mathscr{E}\left(N, \overline{\mathbf{R}}^{n}\right) \rightarrow \mathscr{E}\left(\bar{B}^{n}, \overline{\mathbf{R}}^{n}\right)
$$

such that for $e \in \mathscr{E}\left(N, \overline{\mathbf{R}}^{n}\right), \varepsilon(e)$ extends $e \mid S^{n-1}$, and $e(N) \subset \varepsilon(e)\left(\bar{B}^{n}\right)$. Moreover, if $e$ is quasiconformal then so is $\varepsilon(e)$. 
6. Consequences. In this section we verify Corollaries 4,5 and 6 and consider two-sided flatness.

Proof of Corollary 4. Assume first that $D$ is quasiconformally equivalent to $B^{n}$. Then $\partial D$ is locally quasiconformally flat in $D$, by 17.20 of [14].

Conversely, by Theorem $1, \partial D$ is quasiconformally flat in $D$, say

$$
h: U \rightarrow[a, 1] \quad(a<1)
$$

is a homeomorphism, where $U$ is a neighbourhood of $\partial D$ in $\bar{D}$, such that $h \mid U \cap D$ is quasiconformal. Let $e:[a, 1] \rightarrow \overline{\mathbf{R}}^{n}$ be the inverse of $h$. Then $e \mid(a, 1)$ is quasiconformal so by Theorem $3 e \mid S^{n-1}$ extends to an embedding $\hat{e}: \bar{B}^{n} \rightarrow \overline{\mathbf{R}}^{n}$ so that $U \subset \hat{e}\left(\bar{B}^{n}\right)$ and $\hat{e} \mid B^{n}$ is quasiconformal. By $[9,18.8], \hat{e}\left(B^{n}\right)=D$, so $\hat{e}$ is a homeomorphism from $\bar{B}^{n}$ to $\bar{D}$ which is quasiconformal on $B^{n}$ to $D$.

Proof of Corollary 5. This result follows from Theorem 2 and Corollary 4 as follows. By Theorem 2, $h$ extends to a quasiconformal embedding $h^{\prime}: U \rightarrow \overline{\mathbf{R}}^{n}$, where $U$ is a neighbourhood of $\partial D$ in $\overline{\mathbf{R}}^{n}$. Let $E$ be an ellipsoid for which $\bar{B}^{n-1} \subset E \subset h^{\prime}(U)$. Since $E$ can be mapped onto $B^{n}$ by a linear quasiconformal mapping, it follows that the exterior of $h^{-1}(E)$ has a quasiconformally flat boundary; hence by Corollary $4, h^{\prime} \mid \partial h^{-1}(E)$ can be extended quasiconformally as a mapping $\hat{h}$ of this exterior onto $\overline{\mathbf{R}}^{n}-E$. The required quasiconformal homeomorphism $g$ of $\overline{\mathbf{R}}^{n}$ extending $h$ is given by

$$
g(x)= \begin{cases}h^{\prime}(x) & \text { if } h^{\prime}(x) \in E, \\ \hat{h}(x) & \text { otherwise. }\end{cases}
$$

Proof of Corollary 6. We extend $e$ over each of the complementary domains as in the proof of the "if" part of Corollary 4. The resulting extension $\hat{e}: \overline{\mathbf{R}}^{n} \rightarrow \overline{\mathbf{R}}^{n}$ must be surjective by $[9,18.8]$, so $\hat{e}$ is a homeomorphism of $\overline{\mathbf{R}}^{n}$. Since $S^{n-1}$ is of finite $(n-1)$-Lebesgue measure, by [14, 35.1], $\hat{e}$ is quasiconformal on $\overline{\mathbf{R}}^{n}$.

Two-sided flatness. Suppose $D$ is a Jordan domain in $\overline{\mathbf{R}}^{n}$ and that $h: \partial D \rightarrow S^{n-1}$ is a homeomorphism, that $a$ and $b$ are real numbers with $0<a<1<b$, and that there is a cover $\mathscr{U}$ of $\partial D$ by open subsets of $\overline{\mathbf{R}}^{n}$ so that for $U \in \mathscr{U}$, the map $h \mid U \cap \partial D$ extends to a quasiconformal homeomorphism

$$
h_{U}: U \rightarrow\left\{r z \in \mathbf{R}^{n} \mid a<r<b \text { and } z \in h(U \cap \partial D)\right\} .
$$

Thus $h$ and $\partial D$ are locally quasiconformally flat on both sides: we might say that $h$ and $\partial D$ are locally quasiconformally flat in $\overline{\mathbf{R}}^{n}$, with an obvious meaning for " $h$ and $\partial D$ are quasiconformally flat in $\overline{\mathbf{R}}^{n}$ ".

Corollary 6 shows us that local quasiconformal flatness in $\overline{\mathbf{R}}^{n}$ implies quasiconformal flatness in $\overline{\mathbf{R}}^{n}$; one treats each side separately and then combines the resulting extensions. The same idea carries over to certain non-Jordan domains as considered in $\S 4$. 


\title{
References
}

[1] Brakes, W. R.: An improved version of the non-compact weak canonical Schoenflies theorem. - Trans. Amer. Math. Soc. 213, 1975, 61-69.

[2] Brown, M.: A proof of the generalised Schoenflies theorem. - Bull. Amer. Math. Soc. 66, 1960, 74-76.

[3] Connelly, R.: A new proof of Brown's collaring theorem. - Proc. Amer. Math. Soc. 27, 1971, 180-182.

[4] Gauld, D. B.: The canonical Schoenflies theorem. - Ibid. 27, 1971, 603-612.

[5] Gauld, D. B.: Local contractibility of spaces of homeomorphisms. - Compositio. Math. 32, 1976, 3-11.

[6] Gehring, F. W.: Extension theorems for quasiconformal mappings in $n$-space. - J. Analyse Math. 19, 1967, 149-169.

[7] Gehring, F. W.: Quasiconformal mappings of slit domains in three space. - J. Math. Mech. $18,1969,689-704$.

[8] Golubitsky, M., and V. Guillemin: Stable mappings and their singularities. - Springer-Verlag, Berlin-Heidelberg-New York, 1973.

[9] GreenberG, M. J.: Lectures on algebraic topology. - W. A. Benjamin, Inc., New YorkAmsterdam, 1967.

[10] Mazur, B. C.: On embeddings of spheres. - Acta Math. 105, 1961, 1-17.

[11] Näkki, Raimo, and M. K. Vamanamurthy: Quasiconformal disks in 3-space. - Ann. Acad. Sci. Fenn. Ser. A I 535, 1973, 1-10.

[12] Rushing, T. B.: Topological embeddings. - Academic Press, New York, 1973.

[13] Siebenmann, L. C.: Deformations of homeomorphisms on stratified sets. - Comment. Math. Helv. 47, 1972, 123-163.

[14] VÄISÄLÄ, J.: Lectures on $n$-dimensional quasiconformal mappings. - Lecture Notes in Mathematics 229, Springer-Verlag, Berlin-Heidelberg-New York, 1971.

\author{
University of Auckland \\ Department of Mathematics \\ Auckland
}

New Zealand

Received 23 August 1976 